\title{
Actions entreprises pour la réduction des doses subies par les travailleurs des centrales PWR
}

\author{
P. JEANSON (*) \\ (Manuscrit reçu le 26 janvier 1978)
}

\begin{abstract}
RÉSUMÉ
La réduction des doses subies par les personnes travaillant dans les"centrales nucléaires est un objectif permanent d'Électricité de France. De nombreuses actions sont entreprises simultanément; certaines d'entre-elles sont menées en collaboration avec le C.E.A. Les études théoriques portent essentiellement sur la formation et le transfert des produits de corrosion et de fission dans le circuit primaire des réacteurs à eau pressurisée ainsi que sur les moyens propres à réduire l'activité déposée. Les études pratiques portent sur la conception des matériels ou des locaux et sur leurs règles d'installation. Elles portent également sur les conditions d'entretien de tous les composants; pour les plus importants, des études d'outillage sont entreprises. Enfin, l'étude et la réalisation d'une dosimétrie journalière devraient permettre de mieux connaître la répartition des doses collectives et, par suite, de diriger judicieusement les actions à entreprendre.
\end{abstract}

\begin{abstract}
The dose limitation for workers in nuclear power plants is a permanent aim of Électricité de France. Many ways are followed at the same time to reach it, some of them in collaboration with C.E.A. Theoretical studies concern mostly sources and transfers of corrosion and fission products in the primary circuit of pressurized water reactors as well as the means for reducing the deposited activity. Practical studies concern design problems for equipment and buildings. They try to specify rules for these purposes. They also concern maintenance conditions of all components; for the most important components, special tools are designed. At last, studies and experimentation of daily dosimetry should bring a better knowledge of the distribution of collective doses and, subsequently, should give a better idea of the actions to be undertaken.
\end{abstract}

(*) Département de Radioprotection. Service de la Production thermique d'Électricité de France, 3, rue de Messine, 75008 Paris.

RADIOPROTECTION, VOL. $13-\mathrm{N}^{\circ} 3$ 


\section{INTRODUCTION}

La réduction des doses subies par le personnel des centrales nucléaires est un souci permanent d'Électricité de France. L'effort entrepris dans ce domaine est très vaste du fait des nombreuses directions dans lesquelles il s'exerce et de l'importance des moyens mis en œuvre. Il est justifié par l'ampleur du programme nucléaire français et par le souci de maintenir les doses des travailleurs aussi bas qu'il est raisonnablement possible. Cet article tente de faire une synthèse des principales actions menées dans ce domaine sans toutefois traiter le cas des situations accidentelles.

La réduction des doses subies par le personnel nécessite une connaissance approfondie des mécanismes de formation des sources actives qui en sont responsables afin de mettre en évidence les paramètres sur lesquels il est possible d'agir pour en réduire l'activité. D'autre part, pour une activité donnée des sources, la réduction des doses s'opère par une réduction du temps d'exposition aux rayonnements ou par une réduction du débit de dose (usage d'écrans absorbants ou travail à distance) et, dans une certaine mesure, par la décontamination préalable des circuits actifs.

\section{2. ÉTUDE DES SOURCES ACTIVES}

Les sources peuvent être classées, selon leur origine ou leur mode de formation, en deux catégories :

- les produits de fission formés dans le combustible qui, lui-même, émet des neutrons;

- les produits d'activation formés par le flux neutronique issu du combustible.

Les produits de fission (dans le cas de ruptures de gaines) et certains produits d'activation sont véhiculés par le fluide primaire. Une partie d'entre eux se dépose et occasionne ainsi, sur l'ensemble des circuits véhiculant des fluides actifs, des dépôts dont l'activité devient rapidement supérieure à celle de l'eau. D'autre part, les fuites inévitables des circuits peuvent entraîner une contamination des sols et de l'air des locaux. Cette observation de la nature physique des sources ou de leur emplacement géographique permet de distinguer plusieurs origines aux doses subies par le personnel : combustible, air des locaux, structures métalliques activées, fluides, dépôts sur la paroi interne des tuyauteries, etc.

Lorsque l'on estime l'importance relative de chacune d'elles, il apparaît que 85 p. cent des doses subies par le personnel sont dues aux dépôts formés sur la paroi interne des tuyauteries. Le principal responsable est le ${ }^{60} \mathrm{Co}$ qui, à lui seul, en induit 65 p. cent (en l'absence de ruptures de gaines). Ceci montre l'importance de l'étude des sources afin de mettre en évidence les points sur lesquels l'effort doit porter en priorité et explique d'autre part pourquoi l'étude de la réduction des dépôts actifs occupe une place prioritaire. 


\subsection{DÉPÔTS DE PRODUITS DE CORROSION}

La formation et le transfert des produits de corrosion activés dans le circuit primaire des réacteurs PWR constitue la base de ce problème. Cette étude, principalement menée au Commissariat à l'Énergie Atomique, doit aboutir à la mise au point d'un code de calcul (PACTOLE) permettant de prévoir l'évolution dans le temps de l'activité surfacique des dépôts, connaissant la nature des matériaux du circuit primaire et les conditions d'exploitation. La comparaison périodique entre les résultats de calcul et les mesures sur des réacteurs en service permet d'affiner régulièrement ce code dont les résultats sont déjà satisfaisants.

La solubilité, paramètre très important dans le transfert des produits de corrosion, est à l'étude dans des autoclaves en titane. L'étude sera poursuivie sur la boucle Eldorado dans les conditions de fonctionnement d'un réacteur PWR.

Les phénomènes de formation des dépôts sont extrêmement complexes. Des études expérimentales sur des boucles reproduisant les conditions réelles de fonctionnement permettent d'analyser ce processus et d'étudier, en particulier, l'influence de la teneur en lithium dans l'eau, les autres paramètres étant constants (teneur en bore et température).

L'activation des produits de corrosion est due principalement à leur dépôt sur les éléments combustibles, suivi d'un relâchement par érosion ou solubilisation. La connaissance de ce phénomène a une importance particulière puisqu'il est à l'origine de l'activité des dépôts. Des mesures sur des éléments combustibles de réacteurs américains ont été effectuées par Westinghouse. Elles mettent en évidence la répartition inégale de ces dépôts le long des éléments combustibles; elles permettent de mieux comprendre les phénomènes en jeu. En France, les crayons amovibles des éléments combustibles de la centrale de Tihange seront examinés dans le même but. D'autre part, des recherches sont effectuées sur la boucle Bihan (C.E.A.) qui permet d'étudier les dépôts de produits de corrosion sur un élément combustible simulé.

Parallèlement à ces études, des mesures de débit de dose $\gamma$ sur l'ensemble des matériels actifs sont effectuées périodiquement dans les centrales de Chooz et de Tihange. Elles permettent de connaître le niveau d'activité des dépôts dans différents circuits et de suivre leur évolution dans le temps. Ces mesures sont particulièrement utiles pour la conception des nouveaux équipements afin de prendre en compte les impératifs de la protection du personnel dès l'implantation du matériel.

En complément, des prélèvements de dépôts analysés en spectrométrie $\gamma$ et $\alpha$ donnent des informations précises sur la nature des radioéléments déposés et leur activité surfacique. Ces mesures complètent les mesures effectuées au contact des tuyauteries par spectrométrie $\gamma$ directe, ces dernières étant imprécises pour les émetteurs $\gamma$ de faible énergie.

\subsection{DÉPÔTS DE PRODUITS DE FISSION}

Un programme d'étude entrepris dans le cadre de la Commission mixte E.D.F.-C.E.A. sur la sûreté des réacteurs à eau concerne le niveau de contami- 
nation des circuits par des crayons combustibles présentant des défauts de gaine (expériences Bouffon). Le relâchement des produits de fission du combustible est étudié en simulant différents modes de fonctionnement des tranches (suivi de charge notamment). Les prélèvements de dépôts sur les circuits actifs de centrales en service permettent de connaître la nature et l'activité surfacique des produits de fission déposés.

\subsection{AUTres SOURCES}

Au niveau du combustible, un catalogue des caractéristiques du combustible irradié vient d'être élaboré afin de mieux connaître l'activité contenue dans les assemblages des tranches des paliers 900 et $1300 \mathrm{MW}$ en fonction de leur irradiation et du temps de refroidissement. De même, des études ont été menées pour évaluer l'activité et le rayonnement en provenance des grappes de poison ou de contrôle.

L'ensemble des problèmes de transfert de contamination est examiné à la centrale de Fessenheim par un groupe de travail E.D.F.-C.E.A. Des campagnes de mesures, effectuées par des équipes spécialisées des deux établissements, donnent lieu à une analyse détaillée de l'évolution de la contamination dans les circuits et l'air des locaux de cette centrale. Les résultats permettront d'ajuster les modèles de calcul prévisionnels existants. En particulier, le rôle joué par le circuit de contrôle volumétrique dans la production de produits de corrosion sera évalué d'une façon précise.

Le cas du tritium a donné lieu à une campagne de mesures pendant le premier cycle de la centrale de Tihange pour mieux préciser les sources de production et les voies de transfert. A cet égard, les crayons de poison consommable et les grappes de contrôle sont toujours l'objet d'études particulières.

La source que constitue le fluide des circuits actifs est calculée dans différentes conditions d'exploitation :

- Pour dimensionner les protections biologiques, on calcule une valeur enveloppe de l'activité susceptible de se retrouver dans les circuits; pour les produits de fission par exemple, on admet que la valeur enveloppe résulte d'un taux de rupture de gaine équivalent de $1 \mathrm{p}$. cent associé à la plus faible épuration possible des circuits contaminés.

- Pour estimer avec le plus de réalisme possible l'activité contenue dans les fluides et surtout celle déposée sur les parois des circuits pour les différents régimes de fonctionnement de la tranche, on utilise les résultats de mesures effectuées sur des centrales en service. A court terme, les études menées pour mieux connaître et prévoir les activités déposées devraient permettre de définir ces sources dans le cas de fonctionnement normal des réacteurs.

La source que constitue l'air des locaux contaminables et accessibles quand le réacteur est en service (enceinte de confinement en particulier) est calculée au moyen du code VEGA. Ce code permet d'évaluer avec plus de réalisme les débits de dose régnant dans des locaux, compte tenu d'une source de contamination représentée par la fuite d'un circuit, des dispositifs de filtration en service et des dimensions finies des bâtiments en présence. Ces calculs 
permettent de dimensionner judicieusement les dispositifs d'épuration de l'air pour diverses configurations de fonctionnement en assurant un débit de dose par irradiation externe ou par contamination interne réduit à une valeur convenable.

L'étude des sources telle qu'elle vient d'être décrite précédemment permet une meilleure compréhension des phénomènes physiques. Un de ses objectifs est la détermination des paramètres sur lesquels il serait possible d'agir pour en réduire l'activité.

\section{RÉDUCTION DE L'ACTIVITÉ DES SOURCES}

Les moyens d'action sont, en théorie, assez simples :

- réduction ou suppression des éléments chimiques susceptibles de s'activer sous rayonnement neutronique;

- utilisation de matériaux ayant un faible taux de relâchement;

- réduction des dépôts sur les éléments combustibles;

- utilisation d'une filtration efficace et à grand débit;

- décontamination mécanique ou chimique.

Cependant, l'application pratique de ces règles n'est pas évidente et fait l'objet d'études spécifiques.

\subsection{RÉDUCTION DES ÉLÉMENTS SUSCEPTIBLES DE S'ACTIVER SOUS RAYONNE-} MENT NEUTRONIQUE. ÉTUDE DU TAUX DE RELACHEMENT ET DE REDÉPOSITION

Le principal élément indésirable est le cobalt 59, stable, source de cobalt 60 par activation sous flux neutronique : ${ }_{27}^{59} \mathrm{Co}(n, \gamma){ }_{27}^{60} \mathrm{Co}$. Le cobalt est contenu dans le nickel en tant qu'impureté, il est donc présent dans la plupart des alliages en contact avec l'eau primaire.

Pour en réduire la teneur, une des solutions serait d'effectuer un affinage plus poussé, mais la production serait alors trop limitée pour les besoins. Il ne semble pas techniquement possible, à l'heure actuelle, de diminuer sensiblement cette teneur tout en assurant la même production. Par contre, des mesures ont été prises pour fixer les spécifications concernant la teneur de cette impureté au niveau le plus bas possible compatible avec des productions de fort tonnage.

Une autre solution consiste à utiliser des matériaux ayant une faible teneur en nickel, ce qui réduit d'autant la quantité de cobalt susceptible d'être activé. La recherche de tels matériaux fait actuellement l'objet d'études expérimentales au cours desquelles sont, en particulier, mesurés les taux de relâchement et de redéposition des produits de corrosion, dans les conditions physicochimiques nominales d'une boucle de réacteur PWR. Ces mesures ont pour but de sélectionner parmi les matériaux envisageables ceux qui entraîneraient la plus faible activité des dépôts.

Enfin, il serait probablement souhaitable d'éviter l'utilisation d'alliages tels que le stellite dont les caractéristiques en font un matériau de choix pour

voL. $13-\mathrm{N}^{\circ} 3$ 
les portées de robinetterie, mais dont la teneur en cobalt est voisine de 50 p. cent. Des expérimentations sont prévues pour mesurer son taux de relâchement et, si nécessaire, pour tester des matériaux de remplacement.

\subsection{UTILISATION D'UNE FILTRATION A GRAND DÉBIT}

L'eau du circuit primaire est actuellement traitée à petit débit dansle circuit de contrôle volumétrique au moyen de résines et de filtres. Ce système d'épuration nécessite d'abaisser fortement la pression et la température du fluide $\left(60^{\circ} \mathrm{C}\right.$ environ). Il est donc difficile d'envisager une telle filtration, à grand débit, sans porter un lourd préjudice au rendement du cycle. Or, le débit de filtration actuel est notoirement insuffisant pour limiter l'activité des dépôts. C'est pourquoi des recherches sont entreprises pour réaliser une filtration à la température nominale du fluide et à grand débit. Les recherches portent sur des filtres électromagnétiques ou à lits de graphite.

\subsection{DÉCONTAMINATION MÉCANIQUe ET CHIMIQUE}

La décontamination chimique est utilisée de façon courante pour toutes les pièces dont la taille permet l'immersion dans des bacs. Par contre, la décontamination d'éléments volumineux et fixes tels qu'une boîte à eau de générateur de vapeur ou un circuit primaire complet, ne pourrait pas être réalisée de façon routinière à l'aide de cette technique du fait de la mise en œuvre importante qu'elle nécessite. Cependant, ce type d'intervention peut être rendu nécessaire à la suite d'un incident rendant indispensable une réparation du circuit primaire. E.D.F. étudie avec la Société Turco les matériels à utiliser, les conditions d'exécution pour une décontamination chimique d'une portion du circuit primaire et les répercussions sur la conception des centrales.

Les efforts dans ce domaine portent plus spécifiquement sur des méthodes de décontamination de mise en œuvre suffisamment aisée pour envisager une utilisation périodique sur des matériels pour lesquels les interventions entraînent une dose collective élevée. Actuellement, la technique de décontamination par effet mécanique au moyen d'un jet d'eau à très forte pression est expérimentée pour les boîtes à eau des générateurs de vapeur et les parois de la piscine réacteur.

Une technique particulière de solubilisation des produits de corrosion après l'arrêt du réacteur est étudiée au C.E.A. Après deux tentatives infructueuses à la centrale de Chooz, il semble que les connaissances acquises dans le domaine de la cinétique des phénomènes et des paramètres qui l'influencent, permettent d'envisager à nouveau cette technique comme une voie de recherche intéressante.

La réduction de l'activité des sources ne représente qu'un aspect du problème de la réduction des doses : si l'activité des sources est supposée immuable, il est possible d'agir sur le temps de séjour et le débit de dose sur le lieu de travail. 


\section{RÉDUCTION DU DÉBIT DE DOSE ET DU TEMPS DE SÉJOUR}

Les actions engagées dans ce domaine sont extrêmement diversifiées. Elles portent aussi bien sur la mise en place de protections biologiques que sur l'organisation du travail ou la formation du personnel.

\subsection{Protections BiologiQues}

E.D.F. entreprend, pour chaque centrale, le dimensionnement des protections biologiques de façon que l'exploitation soit possible pour un taux de rupture de gaine équivalent de $1 \mathrm{p}$. cent, en limitant le rayonnement ambiant dans les divers locaux. Ces calculs permettent, en outre, de s'assurer que les circulations routinières empruntent des locaux à très faible débit de dose.

En particulier, une étude systématique des débits de dose liés aux opérations de déchargement, stockage et évacuation du combustible irradié a été effectuée. Cette étude a défini les épaisseurs minimales d'eau et de béton nécessaires en partie courante et dans tous les points singuliers du bâtiment réacteur pour limiter les doses intégrées lors des opérations de manutention du combustible irradié.

En plus de ces études, un travail important est entrepris afin de définir les protections nécessaires pour limiter au maximum le flux de rayonnements sortant de la cuve, réacteur en service. Ce flux est, en effet, susceptible d'introduire un débit de dose non négligeable dans des zones accessibles en marche ou de provoquer une activation des structures proches de la cuve pouvant ainsi induire à l'arrêt des sources de rayonnements complémentaires. Les moyens de calculs utilisés pour ces études ont été validés à partir de résultats de mesures effectuées à la centrale de Tihange et, plus récemment, à la centrale de Fessenheim.

\subsection{Conception et installation du MATÉRIEL}

Des efforts importants sont effectués au niveau de l'installation du matériel afin de réduire les doses pour l'exploitation. La séparation des matériels actifs et inactifs, la séparation des matériels actifs entre eux, la commande à distance de certaines vannes sont, par exemple, des moyens devenus routiniers. En complément, certaines parties des installations sont conçues à l'aide de maquettes d'étude (échelle 1/20) représentant non seulement le gros œuvre et les principaux équipements mais encore les tuyauteries de tout diamètre, chemins de câbles, gaines de ventilation... Ainsi, un examen systématique des conditions d'intervention peut être fait dès le début des études.

Par ailleurs, un recueil de règles d'installation à l'usage des dessinateurs projeteurs est en cours d'élaboration. Ce document précisera les règles à respecter pour l'installation du matériel afin d'assurer les meilleures conditions d'exploitation et d'entretien, en particulier vis-à-vis de la protection des per-

voL. $13-\mathrm{N}^{\circ} 3$ 
sonnes. Ce recueil tiendra compte de l'expérience acquise dans les centrales en service ou en construction au cours de visites systématiques ayant pour but de déceler les anomalies observées en matière de sécurité du personnel et d'y remédier.

\section{3. ÉTUDE DES INTERVENTIONS}

Les analyses détaillées des doses collectives entraînées par les opérations d'entretien font apparaître que ces opérations sont responsables de 60 à 80 p. cent des doses annuelles subies par les travailleurs dans une centrale PWR.

Chaque intervention donne lieu à l'établissement d'une " gamme type » dans laquelle sont décomposées les différentes opérations à effectuer. A chacune de ces opérations sont associés le temps nécessaire pour exécuter le travail, l'outillage et les moyens de manutention nécessaires, le nombre et la qualification des agents, ainsi que la dose collective probable. A l'aide de ces gammes types, le personnel a donc une idée précise du travail à effectuer, ce qui réduit le temps d'intervention et, par conséquent, la dose collective.

Les interventions les plus coûteuses en dose donnent lieu à des études particulières afin de mettre au point un outillage spécialisé permettant soit le travail à distance, soit une réduction du temps d'intervention. C'est ainsi que la totalité des travaux à effectuer dans les boîtes à eau des générateurs de vapeur où le débit de dose varie de 8 à $15 \mathrm{R} / \mathrm{h}$ pourrait, dans un proche avenir, être effectuée de l'extérieur des boîtes à eau. Pour certaines opérations, des maquettes à l'échelle 1 ont été réalisées pour mettre au point la technique d'intervention ou le matériel : c'est le cas, en particulier, des étanchéités des pompes primaires et de la plaque tubulaire des générateurs de vapeur du côté primaire et secondaire. Des maquettes à échelle réduite sont réalisées pour mieux étudier certaines opérations telles que les manutentions dans le bâtiment réacteur.

D'autre part, dans les centrales en exploitation, un effort particulier est entrepris pour mesurer la dose collective entraînée par chaque opération d'entretien. La connaissance de ces doses permet de mieux repérer les opérations sur lequelles l'effort doit porter en priorité et d'apporter les améliorations possibles.

La formation du personnel est un élément primordial pour la réduction des doses. Elle s'opère sur deux plans : la connaissance particulière du travail à effectuer et la connaissance des risques et des moyens de protection. La connaissance précise du travail est obtenue à l'aide des gammes types dont il a été question précédemment. Elles permettent au personnel de gagner du temps sur le chantier. Pour certains travaux particuliers, tels que ceux effectués dans les boîtes à eau des générateurs de vapeur, une maquette à l'échelle 1 permet au personnel de s'entraîner avec l'équipement de protection adéquat. La connaissance générale des risques et des moyens de protection est acquise par le personnel au cours de conférences, de travaux pratiques, de stages et d'entraînement sur des chantiers-écoles. 


\section{CONCLUSIONS}

On a tenté de donner un aperçu général des actions entreprises en insistant sur l'ampleur et la diversité des moyens mis en œuvre pour la réduction des doses subies par le personnel des centrales nucléaires PWR. Certaines actions ont un effet à court terme; il est donc possible d'espérer, dès à présent, des améliorations. Un suivi attentif des nouvelles centrales PWR mises en service permettra d'améliorer nos connaissances dans le but de parfaire les moyens mis en œuvre.

Cependant, il ne semble pas que des progrès spectaculaires puissent être obtenus rapidement. Ces progrès viendront probablement des actions à long terme pour lesquelles l'effort entrepris devra être soutenu. 